\title{
Cuestiones entrelazadas.
}

\section{Inducir desde lo transversal}

\author{
Threaded queries. Inducting from transversality
}

\section{Resumen}

Autores:

Miguel Ángel de la Cova Morillo-Velarde miguelangel.delacova@ gmail.com

Carmen Galán Marín cgalan@us.es

Universidad de Sevilla

España

Recibido:29/Jun/2018 Aceptado: 24/May/2019
E aprendizaje inductivo en arquitectura se ajusta especialmente al conocimiento poliédrico de nuestra disciplina, pero son necesarias metodologías que optimicen los recursos, especialmente en la enseñanza reglada. Se establece un modelo metodológico estructurado mediante una secuencia de actividades y un mapa de contenidos, que convergen en unas preguntas hilvanadas - "threaded queries" - en las que subyacen las diversas problemáticas de la asignatura, de carácter práctico. Las preguntas son concretas, y hacen de mecha para el debate en el aula, pues el interés radica en la toma de conciencia de los objetivos y la implicación de las diversas áreas de conocimiento.

Las preguntas han de ser "tejidas" entre ellas minuciosamente por el profesorado. El estudiante las responderá a partir de ejemplos de arquitectura previamente asignados y seleccionados por los profesores. El aprendizaje se realiza sobre el trabajo y las exposiciones de los estudiantes que, liberados de la autoría, adoptan una posición franca y activa ante la materia. Los profesores han de limitar sus actuaciones, en línea con lo establecido por Finkel (2008) en Dar clase con la boca cerrada. Democracia y autoridad, frente a las herramientas de poder tradicionales de acierto o error, propias de un pensamiento especializado.

Palabras clave: Transversal, aprendizaje inductivo, aprendizaje de la arquitectura.

\section{Abstract:}

Inductive learning in architecture fits especially to the multi-faceted knowledge of our discipline, but methodologies that optimize resources are required, especially in formal education. A structured methodological model is set down through a sequence of activities and a content map, organized through threaded queries in which it underlies the various problems of the subject areas. These queries are tangible: the aim is to spark the debate since the interest lie in awakening about the goals of the course and the involvement of different knowledge areas.

Queries have to be woven carefully by the teaching staff. The student will answer them, from researching architectural examples, which are given previously, previously assigned by professors. Students, in small groups, must compile information concerning these examples, which will be the base of their activity. Their learning is performed on their own work and their public statements, which are extricated from the authorship strain. Thanks to this fact, they set a positive and active attitude. Professors must constrain their speech interventions, as established in Finkel's book (2008) Teaching with your mouth shut. Democracy and authority, versus traditional power skills of carrots and sticks.

Keywords: transversal, inductive learning, architectural learning. 


\section{Introducción}

Peter Zumthor reivindica que lo primero que se ha de transmitir a los estudiantes es "que no se encontrarán ningún maestro que plantee preguntas ante las cuales él sepa de antemano la respuesta" (Zumthor, 2010). Hallar, inducir, circular frente a dirigir, dictar, responder.

Las asignaturas transversales en las que coinciden profesores de varias áreas de conocimiento han de evitar sustentar su estructura en una simple sumatoria acumulativa de especializaciones. Siza señalaba sobre este error curricular que:

se confunde a veces cuál debe ser la preparación del arquitecto. El currículo de la Escuela fomenta la tendencia diversificadora y especializadora. Por el contrario, creo que hay una necesidad de desarrollar sobre todo la capacidad de relacionar entre sí las disciplinas que integran la arquitectura (Siza, 1988 p. 51)

Dicha integración puede producirse en asignaturas con profesorado proveniente de varias áreas de conocimiento, si el profesorado "enseña a observar, a inquirir, incita a la rebusca" (Anasagasti, 1937, p. 106).

Con la intención de ligar diversas áreas de conocimiento, de forma estratégica, se realizó esta experiencia o actividad en la que el profesorado apuesta por un método inductivo, en el que el estudiante o grupo, y finalmente la clase entera, se enfrenta a una serie de debates explicativos a partir de ejemplos de arquitectura. Sobre ellos se rastrearán los problemas y parámetros que son los propios del ejercicio de curso, en un procedimiento en el que el profesor se mantiene en silencio, sin dirigir la situación. Sus intervenciones acompañan observaciones puntuales para evitar excesivas derivas de la sesión.

Las preguntas indagan en cuestiones muy tangibles y directas -qué, cómo, por qué, para qué- pero con una enorme carga potencial o discursiva. Las mismas preguntas dirigidas a diferentes arquitecturas provocan discusiones que permiten al estudiante inducir un conocimiento, con cierto éxito a la vista del "ciclo de mejora" realizado mediante encuestas anónimas entre el alumnado antes y después de la experiencia.

El presente artículo referirá en un primer apartado los fundamentos teóricos en los que se cimienta esta propuesta metodológica realizada en una asignatura de claro carácter transversal, por sus contenidos y por los perfiles del profesorado, dentro del seno de la enseñanza reglada obligatoria de la Facultad de arquitectura de Sevilla. Quedan recogidos en él los fundamentos que caracterizan la experiencia: el tiempo como límite, la evaluación o más bien su sustitución por la valoración global de colectivo, y la activación del estudiante siguiendo los planteamientos de Finkel en torno al liderazgo del aula.

Seguidamente, se establecen las herramientas dirigidas al objetivo de "aprender a aprender" (Muñoz Cosme, 2008). Se explicará el sistema de "cuestiones entrelazadas", los criterios de elección de los ejemplos o modelos a indagar, basados más en los procedimientos que alumbran la obra arquitectónica referenciada que el resultado final.

Posteriormente, se establecerán los criterios y roles, la actitud durante el tiempo de la aplicación del método por parte del profesorado, en los que priman los principios democráticos que necesitan de la autoridad del profesorado, pero ejercida sin situarse en el "centro de la clase" (Finkel, 2008).

De todo este proceso se obtendrán unos resultados que son de doble naturaleza y objeto de análisis: los resultados de las encuestas del "ciclo de mejora" realizado durante la actividad y, de forma indirecta, los resultados de los trabajos de curso.

Las conclusiones finales confían en la vía transversal de la enseñanza de la arquitectura, mediante procedimientos fundamentados y definidos para tal fin, como es el caso que se presenta: frente a una enseñanza curricular acumulativa y especializada, la deseada transversalidad se logra al dar el espacio al estudiante, a través de herramientas perfiladas y diseñadas desde la autoridad democrática del profesorado. Sus inducciones abren el camino al hilvanado de conocimientos de una forma rigurosa en lo tecnológico y en lo creativo

\section{Cimentando la}

\section{experiencia: fundamentos}

\section{y secuencia de actividades}

Magendzo señalaba tres categorías de concepciones curriculares, la académica, la tecnológica y la reconstructivista (Magendzo 1998). En el caso de la materia sobre la que se plantea este artículo, el compromiso social (reconstructivismo), la valoración del contenido disciplinar (academia) y la eficiencia (tecnología) se entrecruzan. Estas concepciones curriculares han de vehicularse mediante unas herramientas de aprendizaje, que en no pocos casos en los que la transversalidad es obligada por la propia estructura docente de la carrera, se concentran en dos paradigmas: la profesionalización y el modelo de copia. 


\begin{tabular}{|c|c|}
\hline SECUENCIA DE ACTIVIDADES & 4 horas \\
\hline Envío previo de email con los ejemplos a analizar ya asignados a cada uno de las grupos de trabajo & Semana anterior \\
\hline Ilegada a clases. Saludos. Presentación de la actividad & 10 minutos \\
\hline Test previo & 10 minutos \\
\hline Explicación del trabajo en grupos. Comienzo del trabajo en grupos. Entrega de las preguntas encadenadas. & 10 minutos \\
\hline \multirow[t]{2}{*}{ Desarrollo del trabajo en grupos } & 70 minutos \\
\hline & 100 minutos \\
\hline Descanso & 30 minutos \\
\hline Presentación a los demás de los ejemplos analizados por cada grupo & 15 minutos \\
\hline $\begin{array}{l}\text { Puesta en comun de los Resultados de los grupos. Se harán comparativas por cada una de la } \\
\text { preguntas. Se expone por parte de cada portavoz y se complementa con preguntas por parte } \\
\text { de los compañeros en el aula }\end{array}$ & $\begin{array}{l}15 \text { minutos } / p \\
x 7 \text { problemas } \\
105 \text { minutos }\end{array}$ \\
\hline Comentarios generales de cara a la sesión del proximo día y continuación del trabajo & 15 minutos \\
\hline Test final & 10 minutos \\
\hline Total & 4 horas \\
\hline
\end{tabular}

Figura 1: Secuencia de actividades prefijadas para la actividad.

Fuente: Propia.

Tal como señala Linazasoro (1984) "la Escuela, como la Universidad en general, es ámbito de docencia e investigación y no de reproposición ficticia de relaciones profesionales que no existen" dentro de ese ámbito, por lo que los métodos que emulan las estructuras profesionales no son las más apropiadas. Esto no es contrario a la necesidad de cubrir aquellos aspectos curriculares que acrediten las competencias profesionales a la egresión del estudiante.

Respecto al sistema de copia, las fortalezas no son pocas, aunque tampoco las debilidades. Este tipo de planteamiento docente está ligado al concepto de repetición, prácticamente gremial, o a los controles comparativos de corrección, que sirven de meta a la aptitud del estudiante. Como establece Juan Luis Trillo "es preciso confiar, ahora más que nunca, en una enseñanza formativa, sustituyendo enciclopedismo por oficio y aptitud por actitud" (Trillo, 1993).

En el caso que nos ocupa de la asignatura de Taller de Arquitectura 5 de la Escuela Técnica Superior de Arquitectura de Sevilla, el objetivo principal de la materia es la integración de las distintas competencias que debe adquirir el estudiante en el desarrollo de una propuesta arquitectónica relevante, que le ha de acercar a la realidad interdisciplinar que supone el trabajo arquitectónico. Para ello, se tendrá en cuenta el desarrollo de la capacidad crítica y el compromiso con la sociedad, la cultura y las condiciones y requerimientos actuales. Por todo ello, se imparte como una asignatura "coral" donde concurren profesores de las distintas áreas de conocimiento de la carrera de arquitectura: proyectos, urbanismo, historia, construcción, estructuras, cimentaciones e instalaciones.

Se trata eminentemente de una actividad práctica en la que los estudiantes, en pequeños grupos, desarrollan un proyecto de arquitectura, apoyado por los profesores de diversas áreas de conocimiento en sus respectivos campos, comprendiendo la toma de datos, análisis e interpretación, propuesta y desarrollo del proyecto, e integrando los conocimientos y formaciones propios de dichas áreas.

Este aprendizaje a través de la práctica en taller ha de acercar a los estudiantes a la realidad interdisciplinar que supone el trabajo arquitectónico y plantearlo como un proceso integral que requiere de su participación y/o coordinación en todas las fases del mismo, desde las primeras ideas generadoras del proyecto hasta la elaboración de los documentos constructivos detallados.

El equipo docente debe orientar el proceso para hacer reflexionar al estudiante sobre la relación existente y necesaria entre proyecto y construcción. Arquitectura, técnica, tecnología y construcción se constituyen en las principales líneas argumentales de este ejercicio, que ensayará la modificación de metodologías y sistemas de trabajo. Se incide además en el carácter práctico de las asignaturas, incorporando la idea de enseñanza experimental. 
A dicha transversalidad de áreas de conocimiento se le superpone otra: la del idioma. Los grupos de trabajo se conforman con estudiantes hispanohablantes y/o angloparlantes de nivel medio y de procedencias en las que dicho idioma no es el natural. Esto asegura una riqueza en el conocimiento del idioma al trabajar sobre una misma materia y requiere un cierto esfuerzo extra por parte del profesorado que debe transcribir las informaciones al estudiante en ambos idiomas.

\subsection{La estrategia}

En este marco, la experiencia que vamos a desarrollar en este artículo responde a las metodologías de aprendizaje establecidas por Finkel, entre otros autores, que activan el papel del estudiante pero sin llegar a "empoderamiento" total del estudiante: es decir, los profesores aportan la autoridad de su conocimiento en el tránsito de aprendizaje recorrido por el estudiante, evitando digresiones que puedan provocar problemas de ajustes temporales del ejercicio, básicos en un curso de enseñanza oficial.

Los puntos fuertes de este sistema son, parafraseando los capítulos del libro Dar clase con la boca cerrada de Donald L. Finkel (2008):

- "Dejar que hablen los libros". Y cómo llegar hasta ellos a través de herramientas digitales de calidad que aseguran la accesibilidad a material informativo y crítico contrastado.

- "Dejar que hablen los estudiantes". A través de un "entrelazado" de cuestiones de sencilla respuesta en primera instancia, que dan pie a mayores complejidades a través del debate entre los miembros de cada grupo de clase y los distintos grupos a su vez.

- "Indagar juntos". El análisis realizado por el estudiante o grupo de ellos no responde a "recitar" unos contenidos previos que se ejemplifican. Más bien al contrario, partiendo de lo particular, se extraen procedimientos o acciones que son las que el estudiante ensaya, más allá del parecido formal con el ejemplo.

- "El arte de escribir". El estudiante escribe, a mano, sus respuestas. Este hecho prácticamente desaparecido de las aulas permite aún poner en valor las relaciones artesanales de atención a lo hecho que propugnan pensadores como Richard Sennett (2009), o más específicamente dentro de la arquitectura, Juhani Pallasmaa (2012)

Por otro lado, la actividad permite extraer conclusiones del ritmo y aprendizaje de la clase, sin recurrir a las evaluaciones como método de control.

\subsection{El factor sorpresa: ni notas, ni pistas}

Para obtener resultados positivos en una innovación metodológica, el alumnado debe comprobar que, efectivamente, hay algo que cambia en el aula. Esta "prueba de honestidad" del profesorado será toda una sorpresa, que incentiva al estudiante:

-No hay calificaciones del ejercicio. En ningún momento el profesor evalúa el ejercicio de forma oficial.

-Anonimato: Los test realizados en la entrada y salida para contrastar la mejora en el conocimiento del alumnado se realiza mediante seudónimo.

-La información a recabar es sencilla de localizar: No se pretende en ningún momento forzar al estudiante a búsquedas tediosas de informaciones marginales. Muy al contrario, se les facilita unos primeros accesos a partir de los cuales recabar información con fluidez, lo que permite dedicar su tiempo al estudio de dichas informaciones.

-El contenido de la actividad se plantea por "sorpresa", es decir, en el momento que los estudiantes llegan a su clase ordinaria, previamente avisados de que se hará algo "distinto". Esto evita traer "preparado" el ejercicio, y reclama la máxima atención y concentración del alumnado.

Todo esto se estructura gracias a una secuencia de actividades prefijadas por el "coro" de profesores, que a continuación pasamos a describir (Figura 1).

\subsection{Los tiempos}

Como detonante y punto de partida del modelo metodológico propuesto, se realiza el envío de un email previo con una serie de ejemplos a analizar ya asignados a cada uno de los grupos de trabajo, lo que consigue que todos ellos vengan al aula provistos de documentación y conociendo de antemano el objeto sobre el que van a trabajar.

El diagrama de contenidos se muestra en la figura 2. Tras la presentación de la actividad (S), se solicita rellenar bajo seudónimo el test inicial para la valoración del "ciclo de mejora" (T). Estas preguntas se contestan individualmente y responden a criterios generales de las materias implicadas en el curso. Las "cuestiones entrelazadas" (E) no son exactamente las mismas que en el cuestionario inicial, pero están relacionadas. En el aula se distribuye el tiempo entre el trabajo de los estudiantes en grupos (G) y la puesta en común de todos los grupos (C) con una exposición previa del ejemplo analizado por cada uno de ellos. En la puesta en común, guiados por los profesores, se irán planteando las relaciones posibles entre los distintos ejemplos de cara a completar el mapa de contenidos en lo que se refiere a conceptos a manejar por los estudiantes.

Tras un resumen final de las intervenciones por parte de profesorado, señalando aspectos sobre los que continuar reflexionando (CF), se realiza de nuevo el test que servirá de comparativa para el "ciclo de mejora" (T). 


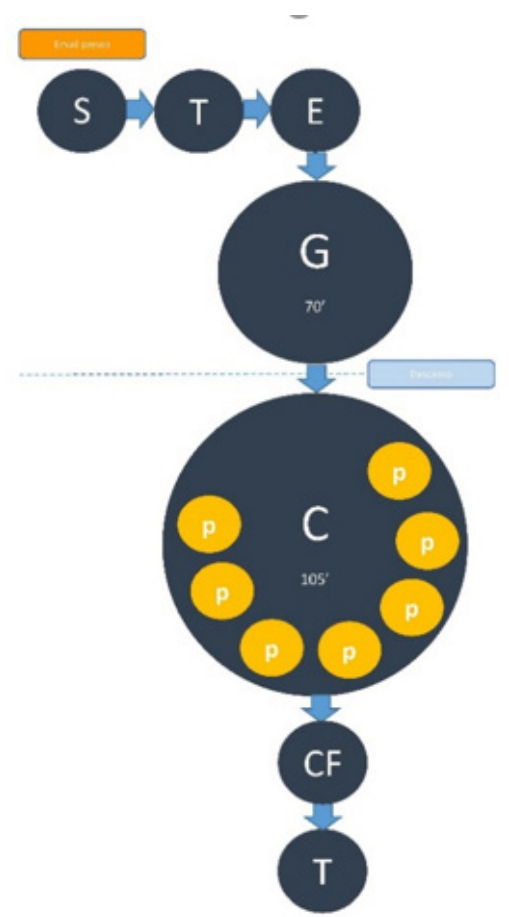

Figura 2: Diagrama de Contenidos. Fuente: Propia

\section{Aprendiendo a aprender: definición de las preguntas y elección de los modelos}

Ya que el profesorado existente va a minimizar sus intervenciones durante el tiempo del ejercicio en clase, el cuestionario de "preguntas entrelazadas" que se les va a entregar adquiere importancia capital.

Las preguntas deben nacer de una puesta en común de los profesores de las materias implicadas en dicha docencia transversal, evitando el protagonismo de alguna de ellas y el "reparto" de cuestiones según la materia.

Tanto las preguntas como los ejemplos propuestos para su análisis inductivo deben partir de un conocimiento de la situación en la que se encuentra el curso. Deben buscarse vías a las que el estudiante pueda acceder por su propia capacidad, pero con cierta sencillez.

\subsection{Las cuestiones entrelazadas: un trabajo colectivo}

Tras realizar el test individualizado y anónimo del inicio, en el que las preguntas están planteadas para inquirir sobre temas genéricos (geometrías, pesos, juntas...), se pasa a la fase del trabajo colectivo. Cada pregunta que habrá de ser respondida a partir del ejemplo estudiado por el grupo, posee diversas trayectorias de conocimiento que se cruzan, en la que cualquiera de ellas puede tomar un carácter preponderante según el perfil de los responsables.

De hecho, este sistema de cruces de áreas en las que las predominancias varían, pero arrastran a su vez el resto de materias implicadas, puede ser un ejemplo de los sistemas "threaded queries" utilizados en programación.

Las preguntas reinciden en la temática ligada al ejercicio planteado en el proyecto docente por De la Cova, Galán y Hernández (2017): la construcción de un pabellón desmontable situado en una plataforma urbana de nueva creación. Dicho pabellón debía ser no solo desmontable sino además ligero y susceptible de rápido montaje, preferiblemente por los propios usuarios. El pabellón había de actuar como reactivador social de un área con carencias en lo que a espacios públicos e infraestructuras sociales se refiere.

Estas cuestiones programáticas planteadas en el proyecto no dejan de ser las mismas genéricas ahora planteadas en el test, pero se convierten en más sencillas de responder en la medida en que lo son desde los ejemplos establecidos, sin preparación previa: puro proyecto de inducción.

El enunciado que indicaba las "preguntas hilvanadas" era el siguiente:

"Sobre el ejemplo previamente indicado, analizar y discutir cada una de las preguntas planteadas":

1. ¿Cuál es la superficie construida aproximada y el programa del edificio analizado? ¿Qué relaciones propone el programa con el entorno inmediato y qué recursos utiliza para ello?

2. ¿Cuáles son los productos utilizados en: Estructura/Cerramiento/Cubierta? ¿Cuál es el peso propio aproximado por metro cuadrado del edificio analizado?

3. Dibuja croquis del detalle constructivo de la envolvente (cerramiento y cubierta) con especificaciones mínimas. Analiza prestaciones de la envolvente en cuanto a estanqueidad y aislamiento.

4. ¿Qué relación hay entre los módulos estructurales y los de la envolvente? (Detalla todas las relaciones que encontréis entre sus dimensiones).

5. ¿Cuál es el sistema estructural de soporte y las dimensiones y módulos de los mismos? Dibuja esquema de la estructura. ¿Cómo se resuelve la rigidización de la estructura frente a acciones horizontales?

6. Analiza las juntas y uniones. ¿Cómo se resuelven los sistemas de unión, estanqueidad, y tolerancias para absorber los errores de montaje...? 
7. Considerando que resulta especialmente relevante construir la estructura con elementos que puedan ser manipulados con medios auxiliares de escasa entidad y los requerimientos de nuestro programa en cuanto a montaje/desmontaje/apilable/autogestión por parte de los vecinos... ¿Qué influencia tiene el proceso constructivo de vuestro ejemplo en la elección del sistema estructural? Discutid si sería posible utilizarlo en vuestro proyecto si se implementasen algunas modificaciones. Proponed las modificaciones requeridas.

Como se observa, las temáticas se entrecruzan entre ellas, arrastrando a las materias, de forma que cada uno de los conceptos requeridos debe y necesita la aportación o el estudio desde las diversas áreas de conocimiento.

\subsection{Elección de modelos: procedimientos vs. formas}

Unos días antes de la actividad, el profesor coordinador de la asignatura coral envía un email anunciando la actividad e instando a los estudiantes (organizados ya en grupo) a recopilar información descriptiva de un modelo que se le ha sido asignado: planimetrías a diversas escalas y que recojan en lo posible soluciones tecnológicas, fotografías del edificio construido y artículos existentes sobre tales ejemplos. Los modelos seleccionados fueron los descritos en las figuras 3 a 7 .

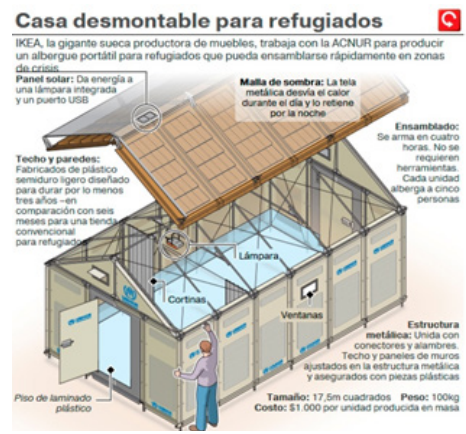

Figura 3: Casa desmontable para refugiados IKEA (2013). Fuente: Recuperado de https://www.graphicnews. com/es/pages/30977/TECNOLOG\%C3\%8DA-Casadesmontable-IKEA-para-refugiados---iGraphic

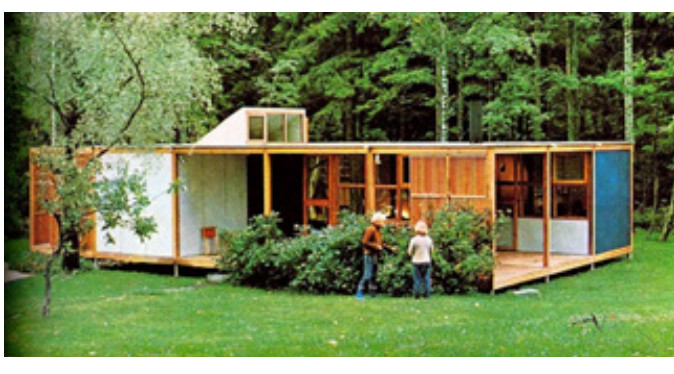

Figura 4: Casa Moduli 225. Pallasmaa y Gullichsen. (1968)

Fuente: Recuperado de https://proyectos4etsa wordpress.com/2011/11/07/sistema-moduli-kristiangullichsen-y-juhani-pallasmaa-finlandia-1968

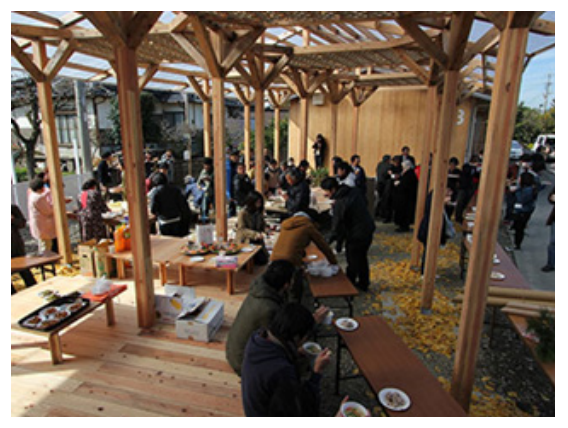

Figura 5: Alojamientos para 'refugiados del maremoto. Shigeru Ban (2016) (2013)

Fuente: Recuperadodehttp://www.shigerubanarchitects. com/works/2016 kumamoto_PHP14

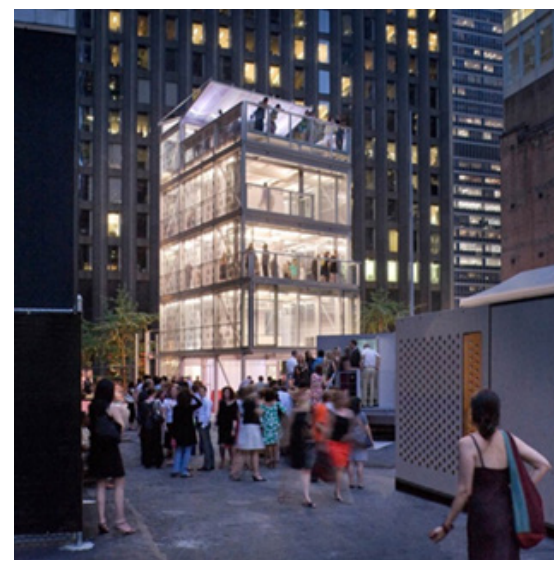

Figura 6: Cellophane house. Kieran Timberlake (2008). Fuente: Recuperado de http://kierantimberlake.com/ pages/view/1

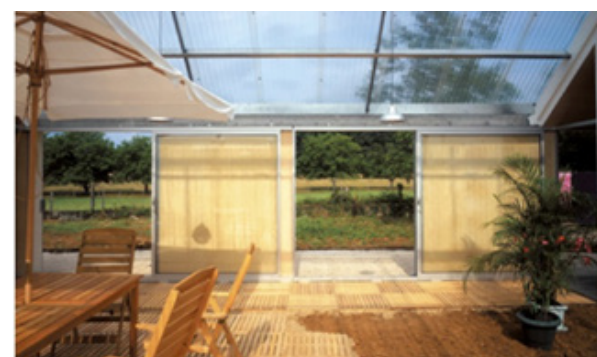

Figura 7: Casa en Dordogne. Lacaton y Vassal (1997). Fuente: Recuperado de https://www.lacatonvassal.com/ index. php?idp=17

En este caso, al estar ya avanzada preliminarmente una primera idea arquitectónica, las memorias o textos de consulta debían centrarse en aspectos más ejecutivos, al igual que con las planimetrías. No obstante, esta misma experiencia se ha realizado con otros agentes (Historia, Expresión Gráfica) y a principios de curso, con buenos resultados también. 
Los ejemplos son relativamente conocidos y esto facilita la recopilación de datos. Para hacerles el camino ágil, se les envían links de páginas webs de las que previamente se ha observado su idoneidad docente, pero sobretodo, páginas web que contengan referencias a bibliografías en papel o digital de relevancia.

Principalmente, en el caso que nos ocupa, se apostó por ejemplos donde los procesos constructivos y materiales varían: cartones, plásticos, maderas, pre-fabricados. Se compromete a los estudiantes en una actividad que les anima a comparar, analizar, evaluar, aplicar y sintetizar de acuerdo con lo propuesto por Ken Bain (2007).

Con ello lo que se pretende es evitar una mala digestión de los aspectos formales de la arquitectura, y favorecer su asimilación a través del proceso de creación y las relaciones entre proyecto y tecnología. Los ejemplos son variados, mayoritariamente del siglo XXI, con el fin de evitar un posicionamiento de "arqueólogo" por parte del estudiante, pero sin dejar atrás alguna obra más lejana en el tiempo, a ser posible no muy conocida. Esta estrategia da la oportunidad de facilitar un entendimiento del valor atemporal de la arquitectura para el aprendizaje, asunto en el que se fracasa cuando solo nos centramos en experiencias recientes o en las ya bendecidas por la historiografía.

\section{Autoridad vs. Poder: dar clase con la boca cerrada}

Al llegar al aula, tras el test inicial de conocimientos, los grupos de estudiantes reciben sus "preguntas hilvanadas". Según la secuencia de actividades, poseen un tiempo para consultar la información que han preparado - la bibliografía que hayan traído (pueden acceder a la biblioteca o internet en el momento que deseen), y contestar a las preguntas por escrito. Finalmente, deberán explicar a sus compañeros las respuestas a cada una de ellas, cambiando el portavoz del grupo en cada una de ellas.

\subsection{El método Finkel: que hablen los estudiantes}

A estas alturas, el alumnado ha cubierto bastantes objetivos: ha trabajado en grupo, ha buscado bibliografía de calidad, ha aprendido cómo llegar a ella a través de la red, y se ha tenido que hacer preguntas a resolver.

Las preguntas, como se ha dicho, permiten un primer acercamiento a la respuesta relativamente rápido, si la información es mínimamente correcta. Esto es bueno puesto que los motores de la actividad han de ser:

-Que los estudiantes se enfrenten y compartan las respuestas específicas al caso que han estudiado, poniendo en relación diversas soluciones o procederes a una misma cuestión: principio de aprendizaje por inducción

-Que el profesor no es el que aporta el conocimiento sino los estudiantes. El docente solo está para evitar derivas o errores importantes no detectados por los propios estudiantes

-Que se está aprendiendo, incluso siendo creativos, a partir de la experiencia de otros. Este hecho libera la idea equivocada de "creatividad ego-centrista" y desterrando la idea del estudio de otras arquitecturas como "tiempo perdido". Este doble hecho tiene un gran aliado en la ausencia del ego-creativo del alumnado ya que, al ser la arquitectura de otros, parece que no existe el peligro de incomodarse con un compañero de clase por hacer una crítica al trabajo realizado.

Durante la experiencia se provoca alto nivel de participación, se suceden comentarios de otros estudiantes y alguno de los profesores presentes en el aula, aclarando cuestiones o poniendo en relación unos ejemplos con otros

Se habla de relaciones de escala, de tipos estructurales, de materiales, de productos... las intervenciones se suceden. El portavoz va cambiando en el grupo en cada pregunta. El diálogo se produce en español e inglés indistintamente en función del estudiante que presenta, lo que permite integrar a todos.

Resulta complicado mantener la limitación de tiempo de cada pregunta, así como la limitación de los conceptos comprendidos en cada una de ellas, cosa lógica por otra parte, pues se les requiere un análisis que puede englobar diversas materias.

Los estudiantes explican sus indagaciones mostrando las imágenes que han recopilado, y también se les solicita que sus dibujos de detalle los lleven a la pizarra, con lo que se provoca una clase muy dinámica donde todos están centrados en lo que estamos trabajando.

Van poco a poco completando un mapa de conceptos con los parámetros analizados en los ejemplos: coordinación de dimensiones, materiales y sistemas constructivos, capacidad de montaje, desmontaje $y$, transporte, peso de las piezas, uniones, juntas, versatilidad del sistema, posibilidades de ampliación..

Finalmente se plantean comentarios generales $y$ recordatorio de la próxima sesión, donde tendrán que tener ya tomadas decisiones respecto a sus propios proyectos.

\subsection{Los profesores, fuera del centro}

¿Y mientras los estudiantes hablan y exponen, cuál es el papel de los profesores? Siguiendo las indicaciones de Finkel, el profesor debe "salir del centro de la clase" (Finkel, 2008, p. 177), tanto física como verbalmente. 
Los profesores han de situarse entre el alumnado, puede que, intercambiando algún comentario coloquial relacionado con la exposición, o simplemente tomando notas. Esta actitud anima al estudiante a hacer lo mismo, desterrando la imagen del estudiante pasivo.

Dicha actitud tiene que ver mucho con el principio de autoridad frente al de poder que establece el autor norteamericano:

Para ser libres de gobernarnos a nosotros mismos, para construir una comunidad democrática (a pequeña o gran escala), debemos ser capaces de coger poder con nuestras propias manos. Pero a la vez debemos respetar las instituciones de autoridad que crean el escenario en el que esperamos ejercer el poder (Finkel, 2008, p. 205).

La autoridad de los profesores se expresa durante la actividad en el control de los tiempos, porque es un garante de la condición democrática e igualitaria del colectivo. Asimismo, son los árbitros que han de saber controlar actitudes inapropiadas ante el colectivo: si los estudiantes no han realizado mínimamente su trabajo, es importante hacerlo ver para que no se pierda la intensidad de la actividad, y esto debe hacerse dando la palabra a otros estudiantes que puedan criticar las respuestas dadas, no incriminando al estudiante.

El otorgar la palabra al estudiante ha de ser el objetivo: inquirirles sobre las respuestas de otros o sobre las propias, pero sin dirigir las respuestas, ha de ser el camino a seguir. En definitiva, el empoderamiento del estudiante no significa la desaparición de la autoridad del profesorado. Probablemente, desde las Vchutemas soviéticas de hace un siglo hasta la AA School en Hooke Park (Figura 8), éste haya sido uno de los fundamentos rara vez expresados que han permitido sistemas de aprendizaje creativos y rigurosos.

\section{Resultados a corto y medio plazo: el final de la actividad y el fin del curso}

Han de extraerse dos tipos de resultados de lo aqu expuesto: una que se extrae del material metodológico utilizado y otra conceptual a partir de los ejercicios realizados por los estudiantes, semanas después de esta experiencia narrada.

En lo que se refiere a las metodologías, el "ciclo de mejora" testado a partir de las encuestas anónimas individuales da unos resultados esperanzadores. Antes y después del ciclo se pasa a los estudiantes el mismo cuestionario con preguntas respecto a los contenidos estudiados en el mismo. En este caso analizaremos a modo de ejemplo la tercera pregunta: ¿Qué productos pueden utilizarse en un edificio desmontable y ligero?

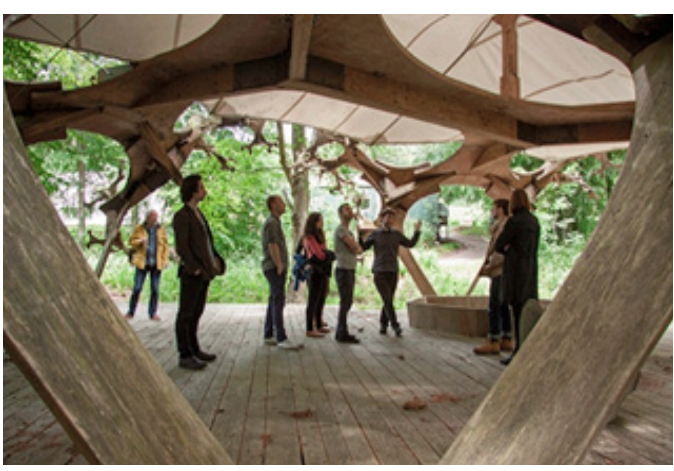

Figura 8: Estudiantes en AA School.

Fuente: Recuperado de ttp://hookepark.aaschool.ac.uk/

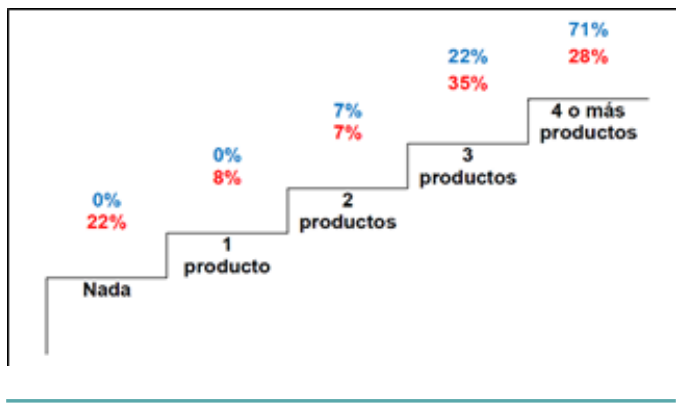

Figura 9: Escalera de conocimientos.

Fuente: Propia

Tal como muestran los gráficos (Figura 9), la escalera de conocimiento mejoró ostensiblemente en una sola jornada. En la escalera inicial (Figura 9, datos en color azul) se aprecia que un $21 \%$ de los estudiantes dejaron sin contestar esta pregunta, aunque una buena parte de ellos conocen varios productos de construcción que podrían utilizar en su proyecto. La escalera final (datos en azul), muestra un claro avance en este sentido, logrando que más de dos tercios de la clase se sitúen en los niveles superiores.

Por otra parte, están los ejercicios que presentaron los estudiantes de su pabellón desmontable. Los ejercicios finales no resultaron en ninguno de los casos "imitaciones" de los modelos. Cada uno de ellos mostró propuestas en las que cualquier parecido formal con los ejemplos era prácticamente casual. Pero sí, por el contrario, se observaron en ellos los resultados del aprendizaje inducido a través de los modelos. La atención al peso de la solución constructiva, sus patrones geométricos, sus posibilidades de manipulación por los propios usuarios incluyendo el montaje-desmontaje, o el potencial de implicar el espacio abierto con el cerrado, encontraron diversas soluciones.

Los proyectos se mostraron mediante maquetas a escala 
1:20 desmontables, presentadas mediante unos archivos GIF que permitían comprobar el proceso de montaje, en un proceder cercano al que se utiliza en la exposición de patentes, para la comprobación de la efectividad del sistema.

Como ejemplo, destacamos el realizado por dos estudiantes (nacionalidades hispano-panameña y turca) que presentaron el pabellón como si de un producto Ikea se tratara, y al que bautizaron "Çiçek", que significa "flor" en turco, aunque parezca sueco. Una arquitectura en la que se registran las esencias de todas esas arquitecturas estudiadas: el uso de la madera de Pallasmaa, el apilamiento de Shigeru Ban, la domesticidad del plástico de Timberlake, e ensamblaje de Lacaton y Vassal y la didáctica de Ikea. Un entrelazado de procesos felizmente construido, aunque sea a 1:20.

\section{Conclusiones: Aprendizaje inductivo}

Se pueden obtener dos conclusiones generales de lo aquí recogido:

-Un método inductivo frente a la acumulación y la especialización: Tal como se observa, tanto los resultados de los trabajos como las escalas de mejora, muestran la superación de los métodos tradicionales. Por un lado, las escalas de mejora en una sola sesión muestran un salto cualitativo de control de la materia que, mediante el sistema acumulativo de datos, hubiera requerido mayores tiempos y probablemente peor asimilación.

En lo que a los trabajos de arquitectura se refiere, los resultados se alejan de la copia académica de las formas, para aprehender las acciones e intereses de la creación arquitectónica.

Dentro del ámbito universitario de formación del arquitecto, una transversalidad consistente en inducir respuestas que requieran la implicación y el conocimiento de diversas áreas de conocimiento al unísono resulta, a tenor de la experiencia mostrada, una vía más eficiente que la simple acumulación de conocimientos y/o una especialización.

Desde el punto de vista metodológico, se ha de insistir en la pertinencia de transferir lo inducido de las sesiones a los casos prácticos que están realizando los estudiantes en el proyecto. Este hecho es clave, pues es el que permite que la experiencia no se convierta simplemente en una actividad informativa puntual, sin más implicación en los resultados.

-Nuevos aprendizajes desde el estudiante: La deseada transversalidad se logra al dar el espacio al estudiante, a través de herramientas perfiladas y diseñadas desde la autoridad democrática del profesorado. Sus inducciones abren el camino al hilvanado de conocimientos, de una forma rigurosa en lo tecnológico y en lo creativo. Actitud frente a aptitud.

Es cierto que este tipo de experiencias encuentran un mejor acomodo en un intervalo temporal algo más amplio, pero también lo es que la docencia oficial reglada permite pocos ajustes en lo que a tiempos se refiere, y que la experiencia ocupa una semana desde que se informa a los estudiantes de la necesidad de recopilación de datos hasta el final de la actividad.

El verdadero objetivo metodológico, no obstante, consiste en que el estudiante valore no solo el "qué" de la cuestión sino sobre todo el "cómo": el savoir-faire, el know-how propio del oficio de arquitectos. Esto es lo que permite que cuando se enfrente a su proyecto, a su creación, lo estudiado no se convierta en un referente puramente formal, sino en una fuente de aprendizaje de procesos de realización, de acciones, en las que hay cabida para el rigor tecnológico, pero, a su vez, para la creatividad artística, tal como mostrara Richard Serra en su "Verb list. 1967-1968" (Figura 10).

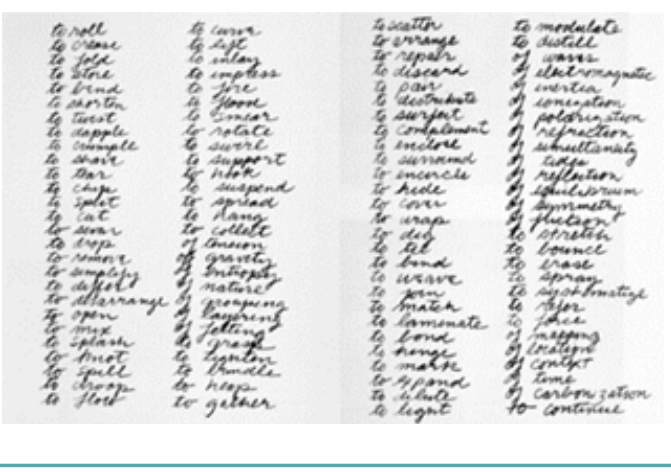

Figura 10: Verb list 1967-1968. Richard Serra. Grafito sobre papel $(25.4 \times 20.3 \mathrm{~cm})$

Fuente:https://www.moma.org/collection/works/152793 


\section{ÇIÇEK}

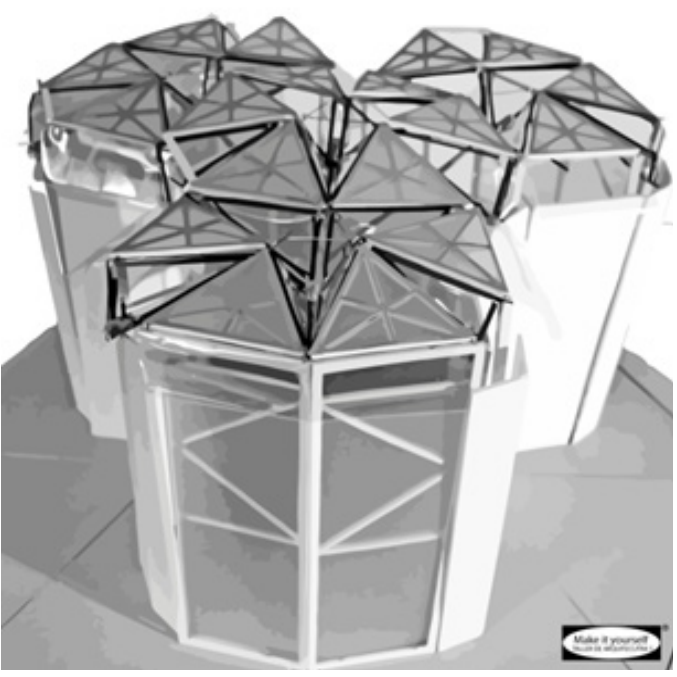

Figura 11: Pabellón Çiçek. Camila Vargas e Ipek Bilge. 2018.

Fuente: Propia.

\section{Agradecimientos}

A Camila Vargas e Ipek Bilge por su consentimiento para la publicación de su trabajo. 


\section{Referencias bibliográficas}

- Anasagasti, T. (1923). Enseñanza de la Arquitectura: cultura moderna técnico artística. Madrid, España: Sucesores de Rivadeneyra.

- De la Cova, Miguel A.; Galán, C.; Hernández, M. (2017). Proyecto docente de la asignatura. Recuperado de http://www.us.es/estudios/grados/plan_233/ asignatura_2330034

- Bain, K. (2007). Lo que hacen los mejores profesores de Universidad. Valencia, España: Publicaciones de la Universitat de Valéncia.

- Finkel, D. (2008). Dar clase con la boca cerrada. Valencia, España: Publicaciones de la Universitat de Valéncia.

- Linazasoro, J.I. (1984). Apuntes para una teoría del proyecto. Valladolid, España: Universidad de Valladolid, Secretariado de Publicaciones.

- Magendzo, A. (1998). El currículum escolar y los objetivos transversales. Pensamiento Educativo, 22.

- Muñoz Cosme, A. (2008). El proyecto de arquitectura. Concepto, proceso y representación. Barcelona, España: Reverté.

- Pallasmaa, J. (2012). Los ojos de la piel: La arquitectura y los sentidos. Barcelona, España: Editorial Gustavo Gili.

- Sennett, R. (2009). El artesano. Madrid, España: Anagrama.

- Siza Vieira, Á. (1988). Enseñanza y Proyecto. Barcelona, Quaderns (176).

- Trillo de Leyva, J. L, (1993). Razones poéticas en arquitectura. Notas sobre la enseñanza de proyectos. Sevilla, España: Escuela Técnica Superior de Arquitectura, Departamento de Proyectos Arquitectónicos.

- Zumthor, P. (2010). Pensar la arquitectura. Barcelona, España: Gustavo Gili. 